\title{
DECLARACIÓN DE HELSINKI: PRINCIPIOS ÉTICOS PARA LA INVESTIGACIÓN MÉDICA SOBRE SUJETOS HUMANOS
}

Análisis de la $5^{\mathrm{a}}$ Reforma, aprobada por la Asamblea General de la Asociación Médica Mundial en octubre del año 2000, en Edimburgo

Respecto del texto aprobado en Somerset West (Sudáfrica) en octubre de 1996

\section{Dr. Jorge Luis Manzini}

Hospital Privado de Comunidad

Programa de Bioética de la Universidad Nacional

Mar del Plata (Argentina)

Correspondencia: Fax 54-223-4990099

E-mail: manzmdq@sinectis.com. 



\title{
DECLARACIÓN DE HELSINKI: PRINCIPIOS ÉTICOS PARA LA INVESTIGACIÓN MÉDICA SOBRE SUJETOS HUMANOS
}

\author{
Análisis de la $5^{\text {a }}$ Reforma, aprobada por la Asamblea General \\ de la Asociación Médica Mundial en octubre del año 2000, en Edimburgo ${ }^{(*)}$ \\ Respecto del texto aprobado en Somerset West (Sudáfrica) en octubre de 1996
}

Dr. Jorge Luis Manzini

\section{Resumen}

Se realiza un análisis detallado de las modificaciones a la Declaración de Helsinki introducidas por la $52^{a}$ Asamblea General de la Asociación Médica Mundial en octubre del año 2000 en Edimburgo.

Luego de una introducción, se efectúa una aproximación general a las reformas de forma y de fondo, seguida por un análisis puntual de cada artículo sustancialmente modificado, comparando además, donde pareció pertinente, las versiones española e inglesa.

Ese desarrollo se hace por la vía de "Comentarios" (XXV en total) al texto transcripto de la versión española circulante en Internet en el sitio www.wma.net/ s/helsinki.html, el 28/11/00.

Las frases o párrafos sujetos a análisis se señalan recurriendo a diferente tipografia, para fragmentos modificados, nuevos, y texto en inglés.

Se analizan los aspectos bioéticos, incluyendo la lectura en "clave de Derechos Humanos", y los aspectos científicos de la investigación sobre seres humanos, considerando la actual perspectiva de la Medicina basada en la Evidencia.

Conclusiones: 1) todas las modificaciones efectuadas en esta reforma de la Declaración apuntan a reforzar la vigencia efectiva de los Derechos Humanos de los sujetos participantes. 2) sería deseable una ulterior revisión del lenguaje de la versión española, que en muchos puntos es ambigua o poco precisa, e inferior en este aspecto a la versión inglesa.

Palabras clave: Declaración de Helsinki, Ética de la investigación, Investigación sobre seres humanos.

\section{Resumo}

Realiza-se uma análise detalhada das modificações feitas à Declaração de Helsinque, introduzidas pela $52^{\mathrm{a}}$ Assambléia Geral da Associação Médica Mundial, em outubro de 2000, em Edimburgo.

Após uma introdução, é efetuada uma aproximação geral às reformas em forma e fundo, seguida de uma análise precisa de cada artigo fundamentalmente modificado, comparando também, onde pareceu pertinente, com as versões espanhola e inglesa.

Esse desenvolvimento é feito através de "Comentários" (XXV em total) ao texto transcrito da versão espanhola circulante pela Internet no site www.net/s/helsinki.html em 28 de novembro de 2000.

As frases ou parágrafos sujeitos a análise são destacadas recorrendo a diferente tipografia, para fragmentos modificados, novos e do texto em inglês.

Os aspectos bioéticos são analisados, incluindo a leitura em "chave dos Direitos Humanos" e os aspectos científicos da pesquisa sobre os seres humanos, considerando a atual perspectiva da Medicina baseada na Evidência.

Conclusões : 1) todas as modificações efetuadas nesta reforma da Declaração visam reforçar a vigência efetiva dos Direitos Humanos dos sujeitos participantes. 2) a revisão da linguagem da versão espanhola deveria passar por uma revisão ulterior, já que em muitos pontos é ambígua ou pouco precisa, sendo considerada inferior, quanto a esse aspecto, em relação à versão inglesa.

Palavras chaves: Declaração de Helsinque, Ética da Pesquisa, Pesquisa sobre seres humanos.

(*) Trabajo presentado para su discusión en la reunión ordinaria del Comité de Etica del Hospital Privado de Comunidad el 13/12/00. Editado en www.aabioetica.org, página de la Asociación Argentina de Bioética, y en www.hva.org.ar, Hospital Virtual Argentino /Proyecto de Bioética. 


\section{Abstract}

This paper consists in a particularised analysis of the Declaration of Helsinki, revised at the $52^{\text {nd }}$ WMA General Assembly at Edinburgh in October 2000.

The framework was as follows:

1) an introduction to the subject 2) a general consideration about formal and substantial aspects of the changes 3 ) a detailed analysis of every modified article, be it a change or an addition; and the comparison, when appropiate, between the Spanish and the English versions of the Declaration.

This plan was developed by means of XXV "Commentaries" interpolated along the transcription of the Declaration, as was available from the WMA website, www.wma.net/s/helsinki.html, on November 28, 2000.

The phrases and paragraphs analysed are identified by means of different printing characters for the modified and new pieces, and the English version.

The analysis was made from a bioethical perspective, especially regarding Human Rights concerns, as well as considering the scientific issues of medical research, including the viewpoint of Evidence based Medicine.

Conclusions: 1) all of the amendments strengthen the Declaration, advocating for higher respect for the Human Rights of research subjects. 2) a further revision of the formal aspects (language) of the Spanish version would be advisable, because of its many punctual weaknesses and ambiguities, as compared with the English version.

Key words: Declaration of Helsinki, Research ethics, Medical research on human beings/subjects,

\section{Résumé}

Une analyse détaillée des modifications à la déclaration d'Helsinki introduites par la $52^{\text {éme }}$ Assemblée Générale de l'Association Mondiale de Médecine a été réalisée en Octobre de l'an 2000 à Édimbourg.

Après une introduction, une aproximation générale aux réformes de forme et de fond a été effectuée, suivie d'une analyse ponctuelle de chaque article modifié de facon substantielle, en faisant en plus la comparaison des versions en espagnol et en anlglais dans les cas pertinents.

Ce développement se fait par la voie de "Commentaires" (XXV au total) tout au long $\mathrm{du}$ texte transcrit de la version en espagnol le 28 novembre de l'an 2000, et qui se trouve en Internet dans le site web: www.wma.net/ helsinki.html.

Les phrases et les paragraphes soumis à l'analyse sont signalés grace à divers typographies, pour les fragments modifiés, nouvaux et pour des textes en anglais.

Les aspects bioéthiques sont analysés, en incluant la lecture en "clef des Droits de l'Homme", et les aspects scientifiques de la recherche sur des êtres humains, en considérant la perspective actuelle de la Médecine fondée sur l'évidence.

Conclusion: 1) Toutes les modifications effectuées dans cette réforme de la Déclaration ont pour objectif renforcer la vigueur effective des Droits de l'Homme des sujets qui y participent. 2) Une révision ultérieure du langage de la version en espagnol serait préférable, puisque dans plusieurs cas elle est ambigüe et peu précise, et aussi inférieure à la version en anglais dans cet aspect.

Mots clés: Déclaration d'Helsinki, Éthique de la recherche, Recherche sur des êtres humains. 
La Declaración de Helsinki, adoptada en la capital finlandesa por la Asamblea General de 1964 de la Asociación Médica Mundial (World Medical Association, WMA), es el documento internacional más importante de regulación de la investigación en seres humanos desde el código de Nürenberg de 1947. La versión anterior es la acordada en Somerset West, Sudáfrica, por la $48^{\mathrm{a}}$ Asamblea General en 1996.

Esta es la quinta vez que se modifica, y ha sido a la vez, la más extensa y profunda de las cinco reformas.

Los borradores estuvieron disponibles para su examen desde 1997, y fueron discutidos en reuniones ad-hoc promovidas por la Asociación, y en sus Asambleas anuales, sin poderse acordar un nuevo texto en 1998 ni en 1999. Se hicieron "amplias consultas a las asociaciones médicas de los distintos países, a grupos representantes de pacientes y a otras partes interesadas" (1). Mucho se publicó a favor y en contra de las reformas propuestas (2-4). Desde abril de 1999 el proyectado texto, ya con muchos cambios, estaba disponible en Internet y provocó un debate particularmente acalorado, porque se temió que varias de las reformas propuestas implicaran "un intento de debilitar los principios éticos del documento y reducir la protección de los participantes en investigaciones médicas, especialmente en países en desarrollo" (1).

Luego de nuevas modificaciones que se le fueron imprimiendo al texto a medida que se iban recibiendo propuestas y eran aceptadas, se llegó a la 52a Asamblea en Edimburgo y se logró consensuar el texto reformado.

En lo formal,

- A la Introducción se le dio el carácter de Parte («A») y se numeraron sus declaraciones, como artículos.

- La Parte «B» (en la versión anterior, «I») mantiene el nombre de "Principios Básicos", pero subsume la anterior Parte
«III» - "Investigación no-terapéutica".

- Hay una Parte «C» que equivale a la antigua «II»-"Investigación médica combinada con la atención médica"-.

- La numeración, que en la versión anterior excluía a la Introducción, como se ha dicho, y empezaba de «1» en cada parte (o sea que había que citar «Art. tal, Parte $t a l$ ), es ahora correlativa, totalizando 32 artículos.

- Algunos párrafos pasaron de una parte a otra, se fundieron, se separaron, etc. Otros fueron modificados y/o ampliados considerablemente. Finalmente, hay alrededor de un $20 \%$ de texto absolutamente nuevo.

- La versión inglesa no difiere sustancialmente de la española, pero hay algunos puntos redactados en forma más precisa y/o taxativa en la primera.

En cuanto a lo sustancial, se puede coincidir con el Dr. Anders Milton, presidente de la Asociación, en que el resultado final ha sido el de fortalecer la Declaración.

Por ejemplo,

- Se aumentan las exigencias para investigaciones hechas sin consentimiento informado, que deben ser la excepción.

- Debe ser esperable que las poblaciones sobre las que se desarrolla la investigación se beneficien de ella.

- Se deben declarar los conflictos de interés.

- Se refuerza el derecho de cada participante en una investigación, aún si le toca integrar el grupo de control, a disponer del tratamiento disponible mejor probado (con lo que el uso de placebo queda reservado para el caso en que no haya ningún tratamiento disponible para la situación que se va a investigar).

- Los participantes en la investigación deberán tener, además, acceso al mejor tratamiento disponible, identificado por la investigación, después de que esta 
termine, regla del "best standard" y no, por ejemplo, del "local standard"(2), como había sido propuesto (5).

A continuación se inserta una copia de la Declaración en idioma español en la que se han ido marcando los párrafos notablemente modificados, sea por redacción, amplitud o ubicación; párrafos nuevos; y palabras o frases de la versión inglesa, cuando la diferencia se consideró digna de mención. Se tomaron para el análisis las versiones inglesa y española circulantes en Internet, sitio de la Asociación Médica Mundial (WMA), www.wma.net/s/helsinki.html, el 28/11/00. Donde pareció pertinente, se efectúan comentarios puntuales.

Se identifican las modificaciones de la siguiente forma:

- En cursiva subrayada, los párrafos modificados.

- En cursiva negrita, los párrafos nuevos.

- En negrita, la expresión correspondiente a la versión inglesa.

\section{A. Introducción}

1. La Asociación Médica Mundial ha promulgado la Declaración de Helsinki como una propuesta de principios éticos que sirvan para orientar a los médicos y a otras personas que realizan investigación médica en seres humanos. La investigación médica en seres humanos incluye la investigación del material humano o de información identificables.

\section{Comentario I:}

Esto es ampliación de una declaración previa que estaba al final de la Introducción. Se le agregó la especificación de " el material humano o de información identificables”.
2. El deber del médico es promover y velar por la salud de las personas. Los conocimientos y la conciencia del médico han de subordinarse al cumplimiento de ese deber.

3. La Declaración de Ginebra de la Asociación Médica Mundial vincula al médico con la fórmula "velar solícitamente $\mathrm{y}$ ante todo por la salud de mi paciente", y el Código Internacional de Ética Médica afirma que: "El médico debe actuar solamente en el interés del paciente al proporcionar atención médica que pueda tener el efecto de debilitar la condición mental y física del paciente".

4. El progreso de la medicina se basa en la investigación, la cual, en último término, tiene que recurrir muchas veces a la experimentación en seres humanos.

5. En investigación médica en seres humanos ( on human subjects), la preocupación por el bienestar de los seres humanos (of the human subject) debe tener siempre primacía sobre los intereses de la ciencia y de la sociedad.

\section{Comentario II:}

La expresión inglesa of the human subject, en singular la segunda vez, aclara con más precisión que se refiere a los sujetos humanos implicados en esa investigación particular.

6. El propósito principal de la investigación médica en seres humanos es mejorar los procedimientos preventivos, diagnósticos y terapéuticos, y también comprender la etiología y patogenia de las enfermedades. Incluso, los mejores métodos preventivos, diagnósticos y terapéuticos disponibles (best proven) deben ponerse a prueba continuamente a través de la investigación para que sean eficaces, efectivos, accesibles $y$ de calidad (must continuously be challenged through research for their effectiveness, efficiency, accesibility and quality). 


\section{Comentario III:}

Párrafo nuevo que reconoce que la mayoría de los métodos en uso no tienen eficacia comprobada. Sería conveniente un análisis lingüístico respecto de cómo traducir "proven". ¿Se refiere a probados, en el sentido de examinar, ensayar (el verbo más usado en este sentido en inglés es "to test"), o a comprobados? Según se decida será entonces, mejor probados, o mejor comprobados. Sabemos que en la discusión previa se quería utilizar la expresión "de eficacia comprobada" (1), lo cual remite a la "Medicina basada en la Evidencia", con la limitación señalada de que la mayoría de los métodos en uso no tiene eficacia probada, y recién ahora se está pretendiendo probarlos, con las dificultades consiguientes (6). Si se quisiera llegar a Evidencia de tipo I (ensayos controlados), debería probarse un procedimiento en uso, de eficacia no comprobada, frente a un placebo. Con el imprescindible consentimiento informado, ¿cuántos serían los voluntarios dispuestos a participar?

La versión inglesa dice "por su efectividad, eficiencia, accesibilidad y calidad". La española, "eficaces, efectivos...”. Eficacia y eficiencia no es lo mismo:

Eficaz, es un procedimiento que logra lo que se esperaba de él, en un contexto experimental.

Eficiente, es un procedimiento eficaz que tiene un costo aceptable.

Efectivo, es un procedimiento que se muestra eficaz en la práctica clínica, lo que tiene que ver con accesibilidad, aceptabilidad, adherencia, etc., por parte de los pacientes.

7. En la práctica de la medicina y de la investigación médica del presente, la mayoría de los procedimientos preventivos, diagnósticos y terapéuticos implican algunos riesgos y costos.
8. La investigación médica está sujeta a normas éticas que sirven para promover el respeto a todos los seres humanos y para proteger su salud y sus derechos individuales. Algunas poblaciones sometidas a la investigación son vulnerables y necesitan protección especial. Se deben reconocer las necesidades particulares de los que tienen desventajas económicas y médicas. También se debe prestar atención especial a los que no pueden otorgar o rechazar el consentimiento por sí mismos, a los que pueden otorgar el consentimiento bajo presión (those who may be subject to give consent under duress), a los que no se beneficiarán personalmente con la investigación y a los que tienen la investigación combinada con la atención médica.

\section{Comentario IV:}

Párrafo nuevo que tiene en cuenta a las poblaciones especiales, vulnerables, más débiles, y los riesgos de coerción más o menos encubierta o disfrazada. Estos asuntos estaban ya contemplados en las International Guidelines de CIOMS-WHO [OMS] de 1993 (7).

La versión inglesa podría traducirse mejor como "aquellos que pudieran ser sometidos a presión para dar su consentimiento".

9. Los investigadores deben conocer los requisitos éticos, legales y jurídicos para la investigación en seres humanos en sus propios países, al igual que los requisitos internacionales vigentes.

No se debe permitir que un requisito ético, legal o jurídico disminuya o elimine cualquiera medida de protección para los seres humanos establecida en esta Declaración.

\section{Comentario V:}

La primera parte del art. 9 contiene 
una ampliación de una declaración previa, que se complementa con la segunda parte, enteramente nueva, en enfatizar la responsabilidad ética y legal del investigador. Además, en consonancia con las modernas tendencias del Derecho Internacional de los Derechos Humanos, pone a la Declaración por encima de las leyes locales, que pueden ser inmorales. La preeminencia de la Declaración debe entenderse sólo como un imperativo moral, no vinculante en sentido jurídico, por no provenir de las Naciones Unidas, un Tratado entre Estados, un Tribunal internacional, etc. (8).

\section{PRINCIPIOS BÁSICOS PARA TODA INVESTIGACIÓN MÉDICA}

10. En la investigación médica, es deber del médico proteger la vida, la salud, la intimidad (privacy) y la dignidad del ser humano.

\section{Comentario VI:}

Esta afirmación estaba en el punto 1 de la Parte III ("Investigación no-terapéutica") de la versión anterior ("proteger la vida y la salud", y la apelación a la defensa de "la intimidad y la dignidad' en el punto 6 de "Principios Básicos", aunque no con las mismas exactas palabras. La versión inglesa dice "privacy" donde la española dice "intimidad". Parece ser que para el Derecho Internacional de los Derechos Humanos, los términos son equivalentes (Rodríguez Fanelli L, comunicación personal, dic. del año 2000).

11. La investigación médica en seres humanos debe conformarse con los principios científicos generalmente aceptados, y debe apoyarse en un profundo conocimiento de la bibliografía científica, en otras fuentes de información pertinen- tes, así como en experimentos de laboratorio correctamente realizados y en animales, cuando sea oportuno.

12. Al investigar, hay que prestar atención adecuada a los factores que puedan perjudicar el medio ambiente. Se debe cuidar también del bienestar de los animales utilizados en los experimentos.

13. El proyecto y el método de todo procedimiento experimental en seres humanos debe formularse claramente en un protocolo experimental. Este debe enviarse, para consideración, comentario, consejo, y cuando sea oportuno, aprobación, a un comité de evaluación ética especialmente designado, que debe ser independiente del investigador, del patrocinador o de cualquier otro tipo de influencia indebida. Se sobreentiende que ese comité independiente debe actuar en conformidad con las leyes y reglamentos vigentes en el país donde se realiza la investigación experimental.

El comité tiene el derecho de controlar los ensayos en curso. El investigador tiene la obligación de proporcionar información del control al comité, en especial sobre todo incidente adverso grave. El investigador también debe presentar al comité, para que la revise, la información sobre financiamiento, patrocinadores, afiliaciones institucionales, otros posibles conflictos de interés e incentivos para las personas del estudio (incentives for subjects).

\section{Comentario VII:}

"Derecho", y no "deber" de controlar. La fórmula propuesta originalmente para esta afirmación era "el derecho y el deber de controlar", lo que produjo mucha resistencia, dado que "muchos comités están aún restringidos en su accionar por falta de recursos", lo que la hacía "irrelevante en cuanto a su aplicación en la práctica" (1).

Aparecen las menciones económicas, 
incluyendo el posible conflicto de intereses del investigador.

Esta es toda una novedad: por un lado, la carrera de los investigadores, por la competencia laboral, académica, etc., les exige determinados resultados en tiempos definidos. Por el otro, ha cambiado la fuente de financiación de los proyectos de investigación; hasta hace unos años, esta provenía básicamente de fondos públicos (Estado, Universidades) y semipúblicos (Fundaciones y otras entidades de bien público), pero actualmente es provista en su mayor parte por capital proveniente de la industria farmacéutica y de tecnología biomédica. Estos factores están cambiando las reglas de juego, desde las normas de la ciencia hacia las del mundo de los negocios $(9,10)$.

La exigencia de mencionar fuentes y condiciones de financiamiento, conflictos de interés, etc., forma parte de la rutina del análisis de protocolos de investigación de un número cada vez mayor de comités, y de los "datos a declarar" para su publicación, en cada vez más revistas prestigiosas.

Por último, "incentives for subjects", como dice el final del artículo en la versión inglesa, no deja dudas de que se trata de incentivos para los sujetos de investigación, lo que no está tan claro en la versión española.

14. El protocolo de la investigación debe hacer referencia siempre a las consideraciones éticas que fueran del caso, y debe indicar que se han observado los principios enunciados en esta Declaración.

15. La investigación médica en seres humanos debe ser llevada a cabo sólo por personas científicamente calificadas y bajo la supervisión de un médico clínicamente competente. La responsabilidad de (for) los seres humanos debe recaer siempre en una persona con capacitación médica, y nunca en los participantes en la investigación, aunque hayan otorgado su consentimiento.

\section{Comentario VIII:}

"For" aclara mejor, que se refiere a la responsabilidad que el investigador tiene sobre los sujetos de investigación a su cargo.

16. Todo proyecto de investigación médica en seres humanos debe ser precedido de una cuidadosa comparación de los riesgos (risks and burdens) calculados con los beneficios previsibles para el individuo o para otros. Esto no impide la participación de voluntarios sanos en la investigación médica. El diseño de todos los estudios debe estar disponible para el público.

\section{Comentario IX:}

La versión inglesa agrega a los riesgos (risks) las cargas (burdens) que implican un concepto más amplio que incluye los sufrimientos, los costos, las molestias, etc., producidas sobre el sujeto, su familia y la sociedad en general; en este caso, por la investigación en cuestión (11).

17. Los médicos deben abstenerse de participar en proyectos de investigación en seres humanos a menos de que estén seguros de que los riesgos inherentes han sido adecuadamente evaluados y de que es posible hacerles frente de manera satisfactoria. Deben suspender el experimento en marcha si observan que los riesgos que implican son más importantes que los beneficios esperados (potential) o si existen pruebas concluyentes de resultados positivos o beneficiosos.

\section{Comentario X:}

Esperados no es lo mismo que po- 
tenciales (potential). Esperados implica una presunción de efectividad que, por tratarse de un ensayo clínico, no puede anticiparse. Esto podría, en cambio, inducir indebidamente al sujeto a participar.

18. La investigación médica en seres humanos sólo debe realizarse cuando la importancia de su objetivo es mayor que el riesgo inherente y los costos (burdens) para el individuo. Esto es especialmente importante cuando los seres humanos son voluntarios sanos.

\section{Comentario XI:}

En este caso la diferencia es entre cargas y costos (en español dice costos, en inglés burdens). Tanto en Economía de la Salud, como en Bioética, costos se refiere sólo al costo económico, y cargas tiene el significado más amplio que se apuntó en el Comentario IX.

\section{La investigación médica sólo se justifi-} ca si existen posibilidades razonables de que la población, sobre la que la investigación se realiza, podrá beneficiarse de sus resultados.

\section{Comentario XII:}

Afirmación absolutamente nueva que tiene antecedentes en preocupaciones expresadas en las International Guidelines de CIOMS-WHO citadas en la nota 7. Representan un compromiso de respeto por la dignidad de todos los seres humanos, desalentando la instrumentalización de grupos menos favorecidos, por razones de costos, de mayor laxitud de las regulaciones locales, etc.

20. Para tomar parte en un proyecto de investigación, los individuos deben ser participantes voluntarios e informados.

21. Siempre debe respetarse el derecho de los participantes en la investigación a proteger su integridad. Deben tomarse toda clase de precauciones para resguardar la intimidad (privacy) de los individuos, la confidencialidad de la información del paciente y para reducir al mínimo las consecuencias de la investigación sobre su integridad física y mental y su personalidad.

\section{ComentarioXIII}

Intimidad vs. privacy. Ver Comentario VI.

22. En toda investigación en seres humanos, cada individuo potencial debe recibir información adecuada acerca de los objetivos, métodos, fuentes de financiamiento, posibles conflictos de intereses, afiliaciones institucionales del investigador.

\section{Comentario XIV:}

Ver Comentario VII

Beneficios calculados, riesgos previsibles e incomodidades derivadas del experimento. La persona debe ser informada del derecho de participar o no en la investigación y de retirar su consentimiento en cualquier momento, sin exponerse a represalias. Después de asegurarse de que el individuo ha comprendido la información, el médico debe obtener entonces, preferiblemente por escrito, el consentimiento informado y voluntario de la persona. Si el consentimiento no se puede obtener por escrito, el proceso para obtenerlo debe ser documentado formalmente ante testigos.

23. Al obtener el consentimiento informado para el proyecto de investigación, el médico debe poner especial cuidado cuando el individuo está vinculado con él por una relación de dependencia o si consiente bajo presión. En un caso así, el consentimiento informado debe ser 
obtenido por un médico bien informado que no participe en la investigación y que nada tenga que ver con aquella relación.

24. Cuando la persona sea legalmente incapaz, o inhábil física o mentalmente de otorgar consentimiento, o menor de edad, el investigador debe obtener el consentimiento informado del representante legal y de acuerdo con la ley vigente.

Estos grupos no deben ser incluidos en la investigación a menos que esta sea necesaria para promover la salud de la población representada y esta investigación no pueda realizarse en personas legalmente capaces.

\section{Comentario XV:}

Protección de personas integrantes de grupos vulnerables, ya señalada en los Comentarios IV y XII.

25. Si una persona considerada incompetente por la ley, como es el caso de un menor de edad, es capaz de dar su asentimiento a participar o no en la investigación, el investigador debe obtenerlo, además del consentimiento del representante legal.

\section{Comentario XVI:}

Muy actual agregado que tiene en cuenta la Convención Internacional de los Derechos del Niño, tanto como las concepciones bioéticas acerca de la competencia -más allá de la definición legal- del concepto de menor adulto, etc. (12).

26. La investigación en individuos de los que no se puede obtener consentimiento, incluso por representante o con anterioridad, se debe realizar sólo si la condición física/mental que impide obtener el consentimiento informado es una característica necesaria de la población investigada.

\section{Comentario XVII:}

Valen las consideraciones del Comentario XV.

En nuestro país se debería además requerir la opinión del Asesor de Menores e Incapaces, según prescribe el Art. 59 (también el Art. 494) del Código Civil. Las razones especificas por las que se utilizan participantes en la investigación que no pueden otorgar su consentimiento informado deben ser estipuladas en el protocolo experimental que se presenta para consideración y aprobación del comité de evaluación. El protocolo debe establecer que el consentimiento para mantenerse en la investigación debe obtenerse a la brevedad posible del individuo o de un representante legal.

\section{Comentario XVIII:}

Esto es, debe haber muy buenas razones para obviar el Consentimiento Informado, ésta debe ser la excepción y no la regla, y su aceptación, resuelta por el comité de evaluación.

27. Tanto los autores como los editores tienen obligaciones éticas. Al publicar los resultados de su investigación, el médico está obligado a mantener la exactitud de los datos y resultados.

Se deben publicar tanto los resultados negativos como los positivos o de lo contrario deben estar a la disposición del público (should be published or otherwise available).

\section{Comentario XIX:}

Es una exigencia cada vez más extendida en la comunidad científica independiente, la publicación de todos los trabajos, independientemente de sus resultados favorables o desfavorables, o al menos su disponibilidad en algún tipo de registro único. 
Históricamente es una constante que un trabajo que no confirma su hipótesis, sobre todo si no muestra los resultados favorables que se esperan de un procedimiento de diagnóstico o tratamiento, tiene mucha menos chance de ser publicado, y eso favorece un sesgo que puede ser utilizado con fines no científicos.

Además se considera que la opinión pública tiene derecho a saber qué investigaciones está solventando, y que de esta manera se evitaría también la repetición inútil de investigaciones (13-15).

La versión inglesa es a mi juicio más taxativa en cuanto a la disponibilidad de tales datos para su examen público (should be published or otherwise available).

En la publicación se debe citar la fuente de financiamiento, afiliaciones institucionales y cualquier posible conflicto de intereses.

\section{Comentario XX:}

Ver Comentario VII.

Los informes sobre investigaciones que no se ciñan a los principios descritos en esta Declaración no deben ser aceptados para su publicación.

\section{PRINCIPIOS APLICABLES CUAN- DO LA INVESTIGACIÓN MÉDICA SE COMBINA CON LAATENCIÓN MÉDICA}

28. El médico puede combinar la investigación médica con la atención médica, sólo en la medida en que tal investigación acredite un justificado valor potencial preventivo, diagnóstico o terapéutico. Cuando la investigación médica se combina con la atención médica, las normas adicionales se aplican (additional standards apply) para proteger a los pacientes que participan en la investigación.

\section{Comentario XXI:}

Sería más preciso, "se aplican normas adicionales".

29. Los posibles beneficios, riesgos, costos y eficacia de todo procedimiento nuevo deben ser evaluados mediante su comparación con los mejores métodos preventivos, diagnósticos y terapéuticos existentes. Ello no excluye que pueda usarse un placebo, o ningún tratamiento, en estudios para los que no hay procedimientos preventivos, diagnósticos o terapéuticos probados (proven).

\section{ComentarioXXII:}

Ver Comentario III.

30. Al final de la investigación, todos los pacientes que participan en el estudio deben tener la certeza de que contarán con (should be assured of access to) los mejores métodos preventivos, diagnósticos y terapéuticos probados y existentes (best proven), identificados por el estudio.

\section{ComentarioXXIII:}

Durante el período de discusión del borrador de la Declaración hubo dos posiciones encontradas respecto de esta afirmación, originada sobre todo en el problema de los estudios basados en un país y efectuados en otro, concretamente, los estudios de vacunas y antirretrovirales para la infección por virus de la inmunodeficiencia humana (VIH-sida) en poblaciones africanas con alta prevalencia de infección: una de las posturas era esta, que es la que finalmente triunfó ("'best standard"), y la otra, la que sostenía que los sujetos, terminado el estudio, debían tener acceso al trata- 
miento "standard" para su comunidad ("local standard"), aduciendo injusticia para con el resto de esa comunidad en caso contrario (2).

Hay numerosos antecedentes de estudios financiados por la industria en que, una vez terminado el período de observación, se dejó de proveer la droga a los sujetos de experimentación, independientemente de que se hubiera probado o no su eficacia, de que la necesidad de tratamiento fuera por un tiempo prolongado o permanente, etc.

Esta norma provee una compensación razonable para quienes aceptaron participar de la investigación, aparte de despejar dudas sobre que la misma fuera un estudio "de marketing" disfrazado, etc.

"Tener la certeza de que contarán con... los mejores métodos...identificados por el estudio" como dice en español, ¿implica más protección que "assured of access to..."?

En cuanto a "proven" ver comentario III

31. El médico debe informar cabalmente al paciente los aspectos de la atención que tienen relación con la investigación. La negativa del paciente a participar en una investigación nunca debe perturbar la relación médico-paciente.

32. Cuando los métodos preventivos, diagnósticos o terapéuticos disponibles (proven) (do not exist or) han resultado ineficaces en la atención de un enfermo, el médico, con el consentimiento informado del paciente, puede permitirse usar procedimientos preventivos, diagnósticos y terapéuticos nuevos o no probados (proven), si, a su juicio, ello da alguna esperanza de salvar la vida, restituir la salud o aliviar el sufrimiento.

\section{Comentario XXIV:}

En cuanto a proven, ver comentario
III. En la versión inglesa se agrega "no existen o han sido ineficaces"...

Siempre que sea posible, tales medidas deben ser investigadas a fin de evaluar su seguridad y eficacia. En todos los casos, esa información nueva debe ser registrada y, cuando sea oportuno (appropiate), publicada. Se deben seguir todas las otras normas pertinentes de esta Declaración.

\section{Comentario XXV:}

Este agregado introduce y da valor a la consideración del método científico y de las salvaguardas éticas ("Se deben seguir todas las otras normas pertinentes de esta Declaración"), aún para el análisis del caso individual, como iniciador de una investigación más ambiciosa al respecto, y la conveniencia de compartir ese análisis con la comunidad científica a través de la publicación, en este caso del "case report".

En cuanto a la diferencia entre las versiones española e inglesa, diré que "oportuno" da una idea de terminología estratégica, que apropiado (appropiate) no tiene.

\section{Conclusiones}

1) La Declaración ha mejorado notablemente luego de esta $5^{a}$ Reforma; todas las modificaciones apuntan a preservar mejor los Derechos Humanos de los participantes.

2) Sería deseable una revisión ulterior del lenguaje utilizado para la versión española, que en muchos puntos es ambigua, o menos taxativa, que la versión inglesa.

\section{Agradecimiento}

A mis compañeros del Comité de Ética 
del Hospital Privado de Comunidad, por las observaciones que me aportaron durante la discusión de este trabajo, que enriquecieron el análisis y los comentarios.

\section{Referencias}

1. A fifth amendment for the Declaration of Helsinki. The Lancet 2000; 356: 1123.

2. Levine RJ. The need to revise the Declaration of Helsinki [Sounding Board]. New England Journal of Medicine 1999;341: 527-34.

3. Nicholson RH. If it ain't broke, don't fix it. The Hastings Center Report 2000; 30 (1): 6.

4. Rothman KJ, Michels KB, Baum M. [For and Against] Declaration of Helsinki should be strengthened. British Medical Journal 2000;321: 442-5.

5. Christie B. Doctors revise Declararion of Helsinki. British Medical Journal 2000;321: 913.

6. Culpepper L, Gilbert TT. Evidence and ethics. The Lancet 1999;353: 829-31.

7. Council for International Organizations of Medical Sciences, World Health Organization. International Ethical Guidelines for Biomedical Research Involving Human Subjects. Geneva: CIOMS, WHO; 1993.
8. Bidart Campos GJ. Tratado Elemental de Derecho Constitucional Argentino. ( ed. actualizada) vol. 3. Buenos Aires: Ediar; 1995.

9. Rosenberg SA. Secrecy in medical research. New England Journal of Medicine 1996;334:392-4.

10. Korn D. Conflicts of interest in biomedical research. Journal of the American Medical Association 2000;284: 2234-7.

11. Manzini JL. Bioética paliativa. La Plata: Quirón; 1997.

12. Hospital Privado de Comunidad de Mar del Plata. Comité de Ética. Normativa para el rechazo de transfusiones de sangre por razones religiosas (Testigos de Jehová). Revista del Hospital Privado de Comunidad 1998; 1 (1):59-64.

13. Benach de Rovira J, Tapia JA. Mitos o realidades: a propósito de la publicación de trabajos científicos. Mundo Científico 1996;15 (154): 124-30.

14. Committee on Publication Ethics (COPE). Guidelines on good publication practice. British Medical Journal 1999;319; 853-62.

15. Horton R, Smith R. Time to register randomised trials. The case is now unanswerable. British Medical Journal 1999;319: 865-6. 